\title{
Inflation and brane gases
}

\author{
Robert Brandenberger \\ Department of Physics, Brown University, Providence, Rhode Island 02912, USA \\ Damien A. Easson \\ Department of Physics, Syracuse University, Syracuse, New York 13244-1130, USA \\ and Kavli Institute for Theoretical Physics, University of California, Santa Barbara, California 93106-4030, USA
}

\author{
Anupam Mazumdar \\ CHEP, McGill University, Montréal, QC, Canada H3A $2 T 8$
}

(Received 10 July 2003; published 2 April 2004)

\begin{abstract}
We investigate a new way of realizing a period of cosmological inflation in the context of brane gas cosmology. It is argued that a gas of codimension one branes, out of thermal equilibrium with the rest of the matter, has an equation of state which can-after stabilization of the dilaton-lead to power-law inflation of the bulk. The most promising implementation of this mechanism might be in type IIB superstring theory, with inflation of the three large spatial dimensions triggered by "stabilized embedded 2-branes." Possible applications and problems with this proposal are discussed.
\end{abstract}

DOI: 10.1103/PhysRevD.69.083502

PACS number(s): $98.80 . \mathrm{Cq}$

\section{INTRODUCTION}

There has been a lot of recent interest (see e.g. [1] for a review of various approaches) in the possibility that string theory may provide a new scenario for the very early Universe which can address some of the cosmological questions [2] which the inflationary universe scenario does not answer. In particular, extended objects which appear in string theory may play an important role, either in terms of their impact on the bulk dynamics $[3,4]$, or as hypersurfaces on which the matter fields of the standard model of particle physics are localized (see e.g. [5] for a recent review).

In particular, since inflation is the most successful paradigm for explaining the large size and the overall approximate homogeneity of the Universe [6] and also yields the most successful theory for generating primordial density fluctuations and cosmic microwave background anisotropies (see e.g. [7,8] for reviews), it is of interest to explore whether string theory can provide new (and hopefully better motivated) ways of achieving a phase of cosmological inflation in the very early Universe (see e.g. [9] for a recent review). It is possible that a phase of inflation resulting from string theory can already be seen at the level of the four space-timedimensional supergravity description of the low energy limit of the theory. However, it is also of interest to investigate new ways of obtaining inflation which are tied to intrinsically stringy effects. "Brane inflation" is one possibility, in which the separation $[10,11]$ or the angle [12] between two branes yields the inflaton field (see also [13,14] for related approaches). Another way of obtaining cosmological inflation is via the mechanism of topological inflation $[15,16]$ resulting from the defects which form as a consequence of brane-antibrane annihilation (see e.g. [17] for the basic physics). Yet another possibility is to have inflation in the context of "mirage cosmology," where our space-time is a test brane moving in a curved background space-time [18-20].

In this paper we suggest a new way of obtaining bulk inflation from intrinsically stringy physics. We observe that (in the context of considering stringy matter on a dynamical background field, and after fixing of the dilaton field) the winding modes ${ }^{1}$ of a gas of co-dimension-1 branes (out of thermal equilibrium) have an equation of state which yields power-law inflation, as long as the separation of the branes is much smaller than the Hubble radius. ${ }^{2}$

Since the resulting accelerated expansion of space will blow up the curvature radius of these branes relative to the Hubble radius, in our proposed mechanism inflation will be of finite duration (there is no graceful exit problem), since the accelerated expansion will end once the separation of the branes becomes comparable to the Hubble radius. After inflation ends, the Universe will take on a phase of nonaccelerating dynamics governed by a network of branes with curvature radius comparable to the Hubble radius. This phase must end before the time of nucleosynthesis in order to avoid an overabundance problem analogous to the "domain wall problem" of particle cosmology [22]. We comment on various possibilities to avoid this problem, the most promising of which appears to be to use "stabilized embedded walls" (unstable 2-branes stabilized by plasma effects) to drive inflation.

In the following, we briefly review some of the required background from string theory. Next, we discuss our inflationary solution and study the dynamics after inflation. In the final section, we discuss ways in which our mechanism could fit into a string cosmology scenario of the early Universe, and address some problems of the proposed mechanism.

\footnotetext{
${ }^{1}$ As demonstrated below, all we require is a gas of branes with a curvature radius larger than the Hubble radius.

${ }^{2}$ Note that an interesting but unrelated mechanism by which a thermal gas of winding modes of open strings on D-branes causes inflation of the brane in a very high energy regime of Hagedorn density was suggested in [21].
} 


\section{BACKGROUND}

Besides the perturbative string excitations, the nonperturbative spectrum of string theory includes $D p$ branes [23], hypersurfaces with $p$ space-like and one time-like dimension on which open strings end. There are stable and unstable branes. Stable (BPS) branes couple to gauge fields (coming from the Ramond-Ramond sector) of rank $(p+1)$, where $p$ is odd for type IIB string theory, and $p$ is even for type IIA string theory. There also exist unstable (non-BPS) $D p$-branes with the complementary dimensions, i.e. $p$ odd (even) in type IIA (B) string theory [24,25] (see e.g [26] for a review of stable and unstable branes in string theory). The instability of a non-BPS brane is manifested in terms of the presence of a tachyonic field in its world volume theory.

In the context of cosmology, both stable and unstable branes may play a role. The field theory analogs of stable branes are topological defects. It is well known that defects can play an important role in early Universe cosmology (see e.g. [27-29] for reviews). The field theory analogs of unstable branes are embedded defects (see the following paragraph), and it has recently been realized that these can also be of importance in the early Universe. Under certain circumstances, unstable defects are stabilized by plasma effects [30,31] (see e.g. [32-34] for some recent suggestions of their role in cosmology). Similarly, unstable branes could be stabilized in the early Universe and play a role in cosmology. For some possible applications of unstable branes in cosmology unrelated to our present work see e.g. $[15,35,36]$. In the following, we will call unstable branes which are stabilized by plasma effects "stabilized embedded branes."

Certain conditions on the coupling of brane or bulk fields to the order parameter describing the unstable brane must be satisfied in order for our proposed mechanism to work. Recall [30] that unstable topological defects can be stabilized if after symmetry breaking there are gauge fields (photon field in the case of the electroweak Z-string) which couple asymmetrically to the different components of the order parameter (Higgs field), and these gauge fields are still in thermal equilibrium. We assume that brane and bulk gauge fields play the role of the photon field (in the case of the electroweak Z-string), i.e. they are still in thermal equilibrium after tachyon condensation, and they couple asymmetrically to the different components of the tachyons. It remains to be worked out in which brane scenarios these conditions are met.

The formation of both stable branes and unstable branes stabilized by plasma effects is analogous to the formation of topological defects in cosmology. One scenario is that a network of branes forms after a phase transition in M-theory which occurs as the Universe expands and the temperature drops. By arguments due to Kibble [37] and Zurek [38], such a phase transition will leave behind a random hypersurface network of branes with a microphysical correlation length (the correlation length gives the mean separation and the curvature radius of the brane network). Note that if the phase transition takes place at temperatures significantly lower than the Planck temperature, then the correlation length is much smaller than the Hubble length $H^{-1}$, where $H$ is the Hubble expansion rate. In this case, the effect of the branes on the background geometry can be analyzed in the brane gas approximation, where one averages the matter distribution over a Hubble volume to obtain an average energy density and pressure which determine the evolution of the background cosmology. Note that in this context the branes are out of thermal equilibrium. This is how topological defects emerge in cosmology. Since the nature of nonperturbative string theory is not known, it is not clear that brane gas cosmology admits a phase transition of the above nature as the temperature increases. Another possibility (explored in detail in the context of string gases on a fixed background geometry [3941]) is that as the brane gas density increases, the branes reach a Hagedorn-like [42] phase, in which the energy density of matter is dominated by infinite branes. Such infinite branes, in the context of spatial manifolds which are toroidal, are branes with nonvanishing winding number (see also [43] for work on string thermodynamics in the presence of branes).

To fix a simple example, we consider for the moment all spatial dimensions to be toroidal, and the initial state of matter to be a dense brane gas containing winding modes. Given such an initial dense network of branes out of thermal equilibrium, the correlation length will increase as the scale factor $a(t)$ as the Universe expands. Fluctuations on the branes with wavelength smaller than the Hubble radius will undergo damped oscillatory motion, whereas fluctuation modes with wavelength greater than the Hubble radius will be frozen. Any net translational motion will also be damped out. In the absence of efficient annihilation between modes with opposite winding number, the brane gas will thus approach a gas of static branes whose energy density is dominated by the straight brane contribution. ${ }^{3}$

The equation of state for a gas of straight branes (neglecting possible gauge charges on the branes) was worked out in [44]. Here we briefly review the analysis. The space-time history of a p-dimensional object in a $D=(d+1)$-dimensional space-time with metric $g_{\mu \nu}$ is represented by the world sheet $x^{\mu}=x^{\mu}\left(\zeta^{a}\right)$, where $\mu$ $=0, \ldots, D$ labels the space-time coordinates and $\zeta^{a}$ are the coordinates on the world sheet with $a=0, \ldots, p$. The induced metric on the $p$-brane is $\gamma_{a b}=g_{\mu \nu} x_{, a}^{\mu} x_{, b}^{\nu}$. The action of the brane is the Nambu-Goto action

$$
S_{p}=-\tau_{p} \int d^{p+1} \zeta \sqrt{-\gamma}
$$

where we have assumed a constant dilaton $\varphi$, so that

$$
\tau_{p}=\kappa e^{-\varphi} T_{p}=\kappa T_{p} / g=\kappa(2 \pi)^{-1} g^{-1} \alpha^{\prime-(p+1) / 2} .
$$

Here, $T_{p}$ is the brane tension, $g=e^{\langle\varphi\rangle}$ is the string coupling, and the string scale is given by $\alpha^{\prime}=\ell_{s}^{2}$. We have inserted a

\footnotetext{
${ }^{3}$ Note that the energy density in the many other fields emerging from string theory will redshift faster than the energy density of the winding modes considered here.
} 
factor $\kappa$ which equals 1 for stable branes but can be much smaller than 1 for stabilized embedded branes.

Now consider a gas of $D p$ branes embedded in $d$ spatial dimensions. The energy-momentum tensor is given by variation of the action (1) with respect to the metric $g_{\mu \nu}$

$$
\begin{aligned}
T^{\mu \nu} & =\frac{-2}{\sqrt{-g}} \frac{\delta S}{\delta g_{\mu \nu}} \\
& =\tau_{p} \int d^{p+1} \zeta \delta^{(D)}(x-x(\zeta)) \sqrt{-\gamma} \gamma^{a b} \partial_{a} x^{\mu} \partial_{b} x^{\nu} .
\end{aligned}
$$

For a $D p$-brane gas, Eq. (3) leads to the equation of state [44]

$$
\mathcal{P}_{p}=\left[\frac{p+1}{d} v^{2}-\frac{p}{d}\right] \rho_{p},
$$

where $\mathcal{P}_{p}$ is the pressure and $\rho_{p}$ is the energy density of the gas of $p$ branes. In the relativistic limit $\left(v^{2} \rightarrow 1\right)$, the branes behave as a relativistic fluid with $w \equiv \mathcal{P}_{p} / \rho_{p}=(1 / d)$, while in the nonrelativistic case $\left(v^{2} \rightarrow 0\right)$, we obtain

$$
w=-\frac{p}{d} .
$$

\section{INFLATION FROM BRANE GASES}

We will work in the context of an initially hot, expanding Universe of $d$ spatial dimensions. We start our considerations with a configuration of matter taken to be a dense gas of branes of all dimensions allowed in the underlying theory. The background is described by the Einstein action. This implies that we are assuming the dilaton has been fixed by some mechanism. Under the assumptions discussed in the previous section, the expansion of the early Universe will produce a gas of branes which are straight and static on microphysical scales, and whose equation of state is given by Eq. (5).

The Einstein equations for a $(d+1)$-dimensional Universe can be written as

$$
\begin{aligned}
& \frac{\ddot{a}}{a}=-\frac{8 \pi G_{d+1}}{d(d-1)}\left[(d-2) \rho_{p}+d p_{p}\right], \\
& \frac{\dot{a}^{2}}{a^{2}}=\frac{16 \pi G_{d+1}}{d(d-1)} \rho_{p},
\end{aligned}
$$

where $G_{d+1}$ is the $(d+1)$-dimensional Newton constant.

Since the correlation lengths of branes of different dimensions will be comparable, the matter content of a brane gas will initially be dominated by the branes with the largest value of $p$ (the heaviest of the branes). Neglecting the contribution of the other branes, making use of the equation of state (5) and inserting this into Eq. (6), one can combine Eqs. (6) and (7) to obtain

$$
a(t) \propto t^{2 /(d-p)} .
$$

Thus, provided the power in (8) is greater than 1, i.e. provided that $d=p+1$, we obtain accelerated expansion (power-law inflation). ${ }^{4}$ In three spatial dimensions, a period of inflation is driven by domain walls $(p=2) .^{5}$

Our inflationary scenario has a natural graceful exit mechanism: during the period of accelerated expansion, the correlation length $\xi(t)$ of the defect network increases relative to the Hubble radius. Once $\xi(t)$ is comparable to $H^{-1}(t)=[(d-p) / 2] t$, the brane gas approximation breaks down since the distribution of the branes averaged over a Hubble expansion time becomes inhomogeneous, and inflation will end (see the following section for arguments as to the further cosmological evolution).

The above criterion for the end of inflation allows us to estimate the duration of the inflationary phase. Given our initial conditions, the energy density is dominated by the network of straight $p$ branes. Their energy density at the initial time $t_{i}$ is given by (up to a factor of order unity)

$$
\rho\left(t_{i}\right)=\tau_{p} t_{i}^{p} \frac{t_{i}}{\xi\left(t_{i}\right)} t_{i}^{-d},
$$

where $\tau_{p}$ is the rescaled tension of the $p$ brane, the first two factors on the right hand side of the equation give the energy of a single brane within the Hubble volume, the third factor gives the number of branes per Hubble volume, and the last factor is the inverse of the Hubble volume. The brane tension is given in terms of the string length $\ell_{s}$ and the string coupling constant $g$ by Eq. (2). Making use of the first Friedmann equation (7), and replacing the higher-dimensional gravitational constant $G$ by the corresponding fundamental gravitational length scale $l_{f}$ (defined via $G=\ell_{f}^{d-1}$ ), we obtain (setting $p+1=d$ in order to obtain inflation)

$$
H\left(t_{i}\right)^{2}=\frac{8}{d(d-1) g} \kappa\left(\frac{\ell_{f}}{\ell_{s}}\right)^{d} \frac{1}{\ell_{f} \xi\left(t_{i}\right)} .
$$

The important ratio between Hubble radius and correlation length at time $t_{i}$ is

$$
\frac{H^{-1}\left(t_{i}\right)}{\xi\left(t_{i}\right)}=\left(\frac{d(d-1)}{8}\right)^{1 / 2} g^{1 / 2} \kappa^{-1 / 2}\left(\frac{\ell_{s}}{\ell_{f}}\right)^{d / 2}\left(\frac{\ell_{f}}{\xi\left(t_{i}\right)}\right)^{1 / 2} .
$$

During the period of inflation, the correlation length increases as $a(t)$ whereas the Hubble radius increases only linearly in time. Thus, the correlation length has caught up to the Hubble radius increases at a time $t_{f}$ (the end of inflation) given by

$$
\frac{t_{f}}{t_{i}}=\frac{H^{-1}\left(t_{i}\right)}{\xi\left(t_{i}\right)}
$$

\footnotetext{
${ }^{4}$ Note that this result agrees with what follows from the study of [45] of the late time dynamics of brane gases in M-theory [use $\gamma$ $=3$ and $m_{1}=0$ in their Eq. (40)].

${ }^{5}$ The idea of wall-dominated inflation was first made in [22] (see also [46]).
} 
The ratio of scale factors at $t_{f}$ and $t_{i}$ is the square of this expression. The number $\mathcal{N}$ of e-foldings of inflation is given by

$$
\mathcal{N}=\ln \left(\frac{a\left(t_{f}\right)}{a\left(t_{i}\right)}\right) .
$$

If, as an example, we assume $\xi\left(t_{i}\right)=\ell_{s}$ (motivated by the fact that the initial separation of topological defects after a phase transition is of the order of the inverse of the symmetry breaking scale $[37,29])$, then Eq. (13) gives

$$
\mathcal{N} \simeq \ln \left(g \kappa^{-1}\right)+(d-1) \ln \frac{\ell_{s}}{\ell_{f}} .
$$

Thus, we conclude that it is possible to obtain a sufficient number of e-foldings of inflation to solve the problems of standard big bang cosmology [6] provided that the ratio $\ell_{s} / \ell_{f}$ is large. Working, for example, with $g=\kappa=1, d=3$ and $\ell_{f}^{-1}=10^{19} \mathrm{GeV}$, we require $\ell_{s}^{-1} \leqslant 10^{7} \mathrm{GeV}$ to obtain more than the 55 e-foldings of inflation (required for the scenario to solve the problems of standard big bang cosmology). For stabilized embedded defects with $\kappa^{-1} \gg 1$, the upper bound on the string scale is less severe (i.e. higher).

Note that, from a naive point of view (treating the fluctuations generated in our model like the fluctuations generated in a model of power-law inflation driven by a scalar field with exponential potential), the inflationary period discussed above is not capable of explaining the observed density fluctuations and microwave anisotropies, since the predicted spectral slope $n_{s}$ deviates too much from a scaleinvariant $n_{s}=1$ one. To first order in the power $\alpha=2 /(d$ $-p$ ) which appears in the scale factor $a(t)$ [see (8)] one obtains (see e.g. [47])

$$
n_{s}-1=-\frac{2}{\alpha},
$$

whereas the observed spectral index is now known to be in the range $n_{s}-1=-0.07 \pm 0.03$ [48]. Thus, in order for our scenario to make contact with observed inhomogeneities, we require a different mechanism to produce the primordial fluctuations. We will return to this issue later.

\section{EVOLUTION AFTER INFLATION}

Once the correlation length becomes comparable to the Hubble radius, inflation will end. In particular, at that time the approximation of treating matter as a homogeneous brane gas breaks down, since the typical density fluctuations per Hubble volume, which can be estimated via $\delta E / E$ (where $E$ is the total energy within the Hubble volume, and $\delta E$ is the energy which a single brane contributes), become of order unity.

Once a Hubble volume no longer contains one of the branes responsible for the period of inflation, the dynamics of that volume are determined by whatever matter is left (e.g. the lower-dimensional branes). The volume will undergo nonaccelerating power-law expansion, and now the Hubble radius will increase relative to the correlation length $\xi(t)$ of the $p=d-1$ branes. Thus, the dynamical evolution after the end of inflation is governed by

$$
\xi(t) \sim t
$$

In particular, the energy of the Universe continues to be dominated by these $p=d-1$ branes, leading to a severe domain wall overabundance problem.

Since the energy density of matter other than the $p=d$ -1 branes is suppressed by a power of $e^{\mathcal{N}}$ relative to the energy density of the $p=d-1$ branes, and since the energy density of the $p=d-1$ branes is already quite low, namely given by a typical mass scale

$$
m\left(t_{f}\right)=\rho\left(t_{f}\right)^{1 /(d+1)}
$$

which [making use of Eqs. (9) and (12)] is of the order

$$
\begin{aligned}
m\left(t_{f}\right)= & \left(\frac{4}{\pi d(d-1)}\right)^{1 /(d+1)} g^{-2 /(d+1)} \\
& \times \kappa^{2 /(d+1)} \frac{\ell_{f}}{\ell_{s}}\left(\frac{\ell_{s}}{\ell_{f}}\right)^{2 /(d+1)} \ell_{s}^{-1},
\end{aligned}
$$

there is also a potential problem of how to reheat the Universe.

Thus, it appears that in order to obtain an acceptable late time cosmology, the $p=d-1$ branes must decay, both to solve the domain wall problem and to achieve reheating.

As mentioned at the end of the previous section, the adiabatic fluctuations generated by our model of inflation have the wrong spectral index, namely $n_{s}=0$, and thus cannot explain the observed large-scale structure and microwave anisotropies. Thus, the model needs additional ingredients in order to successfully connect with present observations. Having said this, it is important to realize that the fluctuations generated in our model will not be equivalent to the fluctuations generated in a scalar field toy model which yields the same expansion rate, the reason being that branes (like defects) generated iso-curvature fluctuations. As long as the branes have not decayed, a spectrum of curvature fluctuations which is scale-invariant when measured at Hubble radius crossing will result [49]. At the time of the brane decay, these primordial iso-curvature fluctuations will contribute to an adiabatic mode. The details of this process remain to be studied. In analogy to what can be achieved [50] in the Pre-Big-Bang [51] scenario, where the primordial adiabatic spectrum has the wrong index (namely $n_{s}=4$ [52]) it is possible that other matter fields obtain a scale-invariant spectrum of iso-curvature modes which upon the decay of these fields yields an adiabatic mode via the curvaton mechanism [53-56]. Since the spectrum of our primordial adiabatic mode is red, it is likely that any additional mechanism like the two possibilities we just mentioned will dominate the spectrum on scales of present cosmological interest. 


\section{DISCUSSION}

We have proposed a new mechanism for obtaining bulk inflation in the context of string cosmology. The mechanism is based on a gas of $p=d-1$ branes causing power-law inflation of the $d$ space-dimensional bulk in which the value of the dilaton is fixed. The duration of inflation is automatically finite in our scenario. However, the final state is faced with both a domain wall problem and a reheating problem.

The first issue we wish to focus on in this section is how the domain wall and reheating problems can be resolved. Our idea is that this will occur via the decay of the branes which are responsible for inflation. The decay will release the brane energy into ordinary matter and cause the "temperature" (defined here as the fourth root of the energy density) of the Universe to take on the value given in Eq. (18).

How can brane decay occur? If the branes which are driving inflation are stabilized embedded branes, then the branes will automatically decay once the temperature of the plasma has dropped sufficiently low (see $[30,31]$ ). If the branes are stable, the problem is more severe. One possibility is that eventually the branes and antibranes will annihilate, leaving a state with no long branes. This possibility, although it works for topologically stable branes as well as for branes which are dynamically stable but not topologically stable (no conserved quantum number), seems hard to reconcile with the topological arguments for the existence of long branes (arguments derived in the context of topological defects in field theory-see the reviews [27-29]). Another possibility (suggested first by Seckel [46]) is that holes in the branes nucleate and "eat up" the branes at the speed of light. Since this occurs in the post-inflationary phase, there is no causality obstruction to the local decay of the network of branes within one Hubble volume. However, the local decay is only possible if the walls are not protected by a topological quantum number.

It is important that brane decay occurs sufficiently early such that the Universe has time to homogenize and thermalize sufficiently before the time of recombination (see e.g. [34] for a study of the corresponding constraint on stabilized embedded topological defects). There are also possible constraints from inhomogeneous nucleosynthesis.

The second question addressed in this section is how the mechanism suggested in this paper might fit into a coherent picture of early Universe string cosmology, in particular whether there is any way to connect the proposal made here with the ideas of $[3,4]$ (see also the more recent work of $[57,58]$ ) on how the dynamics of brane winding modes can create a hierarchy of scales between the three spatial dimensions we see and the other spatial dimensions.

In the following, we will take three different starting points corresponding to different corners of the M-theory moduli space and discuss how our proposal might fit in. First, consider $11 D$ supergravity. This theory contains stable M2 and M5 branes. In this context, the dilaton is fixed and the background equations are the Einstein equations. Easther et al. [45] have recently studied the effects of winding modes of M2 branes (on a toroidal Universe where space is $T^{10}$ ) on the dynamics of a homogeneous but anisotropic cosmology and they found that, given initial conditions for which the radii of all the tori are comparable, the dynamics favors three spatial dimensions becoming larger than the other ones (which we call "internal"). Implicitly, the assumption was made that the M5 branes have all annihilated. We will make a stronger assumption, fixing the radion fields corresponding to the internal dimensions, and start with a dense gas of M2 branes. The M2 branes which wrap two of the large spatial dimensions can give rise to inflation by the mechanism we are proposing (M5 branes wrapping three internal dimensions would have the same effect). Since the M2 branes are stable, this scenario has a serious domain wall problem (which, as mentioned at the beginning of this section, can be addressed successfully).

As suggested by [59], it is possible that brane intersections play an important role in M-theory cosmology. This might also lead to new possibilities for brane gas inflation. We leave an investigation of this possibility to future work.

As an orthogonal starting point we consider type IIA string theory. Again, we fix the dilaton and the radion fields by hand. This theory contains stable D2 branes which will generate inflation according to our proposed mechanism. Higher dimensional stable branes for which all but two cycles wrap internal dimensions will contribute to inflation in an analogous way. As in the previous example, we are faced with a serious domain wall problem.

Turning now to type IIB string theory, we first again assume that the dilaton and radion fields have been fixed by some mechanism unrelated to our scenario. In this case, there are stable D1 branes and unstable 2-branes. The unstable 2-branes, provided that they are stabilized by plasma effects early on, can drive a period of inflation by our mechanism. They will decay at late times, and will thus not lead to a domain wall problem. The stable D1 branes will take on a scaling solution like cosmic strings (without dominating the energy density of the Universe) [27-29], and will lead to predictions for fluctuations which are in principle testable. Specifically, these strings yield line discontinuities in the cosmic microwave temperature maps [60]. In order not to conflict with present observational bounds (the most stringent bounds come from the location and narrowness of the first acoustic peak in the spectrum of microwave anisotropies; see e.g. [61] for a recent review on the confrontation between topological defect models and observational constraints), the string scale must be sufficiently low such that the string tension is less than about $10^{28} \mathrm{GeV}^{2}$.

In the context of type IIB string theory we can try to merge the ideas of creating a hierarchy in the scales of spatial dimensions $[3,4]$ with the mechanism for inflation proposed here. We will make use of the fact that odddimensional branes are stable and heavy, but evendimensional branes are unstable. If they are stabilized by plasma effects, it is likely that the core will undergo a "core phase transition" [62] analogous to what occurs for stabilized embedded defects in field theory [30,33,31]. In this case, the effective tension is much smaller than it would be for a stable brane of the same dimension [this was our motivation for introducing the factor $\kappa$ in Eq. (2)]. Let us now begin the time evolution with all spatial dimensions of the 
same size, and the dilaton free. As discussed in [4], all stable higher dimensional branes annihilate, leaving behind the stable D1 branes. These will annihilate only in three spatial dimensions, allowing these to grow, and keeping the others small. Note that if the stabilized embedded branes are much lighter than the stable branes, there still will be stabilized embedded 2-branes after the hierarchy of spatial dimensions develops. Since in the three large dimensions there are no more stable branes present, the stabilized embedded 2-branes will now dominate the dynamics, leading to inflation of these dimensions, and without creating a domain wall problem.

To summarize the discussion of the previous paragraph, it appears that in the context of type IIB it is possible to connect the speculations of $[3,4]$ on the origin of the hierarchy between the sizes of spatial dimensions with late time cos- mology. Key is the mechanism proposed in this paper, namely inflation triggered by a gas of stabilized embedded membranes.

\section{ACKNOWLEDGMENTS}

We wish to thank Cliff Burgess and Rob Myers for useful discussions. At Brown, this work has been supported in part by the U.S. Department of Energy under Contract DE-FG0291ER40688, TASK A. D.E. was supported in part by NSFPHY-0094122 and other funds from Syracuse University and NSF-PHY-99-07949 (KITP, UC Santa Barbara). A.M. acknowledges support from NSERC. A.M. thanks CITA for financial support.
[1] D.A. Easson, Int. J. Mod. Phys. A 16, 4803 (2001).

[2] R.H. Brandenberger, hep-ph/9910410.

[3] R.H. Brandenberger and C. Vafa, Nucl. Phys. B316, 391 (1989).

[4] S. Alexander, R.H. Brandenberger, and D. Easson, Phys. Rev. D 62, 103509 (2000); R. Brandenberger, D.A. Easson, and D. Kimberly, Nucl. Phys. B623, 421 (2002).

[5] P. Brax and C. van de Bruck, Class. Quantum Grav. 20, R201 (2003).

[6] A.H. Guth, Phys. Rev. D 23, 347 (1981).

[7] V.F. Mukhanov, H.A. Feldman, and R.H. Brandenberger, Phys. Rep. 215, 203 (1992).

[8] D.H. Lyth and A. Riotto, Phys. Rep. 314, 1 (1999).

[9] F. Quevedo, Class. Quantum Grav. 19, 5721 (2002).

[10] G.R. Dvali and S.H. Tye, Phys. Lett. B 450, 72 (1999).

[11] C.P. Burgess, M. Majumdar, D. Nolte, F. Quevedo, G. Rajesh, and R.J. Zhang, J. High Energy Phys. 07, 047 (2001); D. Choudhury, D. Ghoshal, D.P. Jatkar, and S. Panda, J. Cosmol. Astropart. Phys. 07, 009 (2003).

[12] C. Herdeiro, S. Hirano, and R. Kallosh, J. High Energy Phys. 12, 027 (2001); J. Garcia-Bellido, R. Rabadan, and F. Zamora, ibid. 01, 036 (2002); M. Gomez-Reino and I. Zavala, ibid. 09, 020 (2002).

[13] K. Dasgupta, C. Herdeiro, S. Hirano, and R. Kallosh, Phys. Rev. D 65, 126002 (2002).

[14] J.H. Brodie and D.A. Easson, J. Cosmol. Astropart. Phys. 12, 004 (2003).

[15] S.H. Alexander, Phys. Rev. D 65, 023507 (2002).

[16] S. Alexander, R. Brandenberger, and M. Rozali, hep-th/0302160.

[17] A. Sen, J. High Energy Phys. 08, 012 (1998).

[18] A. Kehagias and E. Kiritsis, J. High Energy Phys. 11, 022 (1999).

[19] S.H. Alexander, J. High Energy Phys. 11, 017 (2000).

[20] M.F. Parry and D.A. Steer, J. High Energy Phys. 02, 032 (2002); E. Kiritsis, G. Kofinas, N. Tetradis, T.N. Tomaras, and V. Zarikas, ibid. 02, 035 (2003).

[21] S.A. Abel, K. Freese, and I.I. Kogan, J. High Energy Phys. 01, 039 (2001)
[22] Y.B. Zeldovich, I.Y. Kobzarev, and L.B. Okun, Zh. Éksp. Teor. Fiz. 67, 3 (1974) [Sov. Phys. JETP 40, 1 (1974)].

[23] J. Polchinski, String Theory, Vols. I and II (Cambridge University Press, Cambridge, 1998).

[24] A. Sen, J. High Energy Phys. 12, 021 (1998).

[25] E. Witten, J. High Energy Phys. 12, 019 (1998).

[26] A. Sen, hep-th/9904207.

[27] A. Vilenkin and E.P.S. Shellard, Cosmic Strings and Other Topological Defects (Cambridge University Press, Cambridge, 1994).

[28] M.B. Hindmarsh and T.W. Kibble, Rep. Prog. Phys. 58, 477 (1995).

[29] R.H. Brandenberger, Int. J. Mod. Phys. A 9, 2117 (1994).

[30] M. Nagasawa and R.H. Brandenberger, Phys. Lett. B 467, 205 (1999).

[31] M. Nagasawa and R. Brandenberger, Phys. Rev. D 67, 043504 (2003).

[32] R.H. Brandenberger and X.m. Zhang, Phys. Rev. D 59, 081301 (1999).

[33] B. Carter, R.H. Brandenberger, and A.C. Davis, Phys. Rev. D 65, 103520 (2002).

[34] R.H. Brandenberger, B. Carter, and A.C. Davis, Phys. Lett. B 534, 1 (2002).

[35] A. Mazumdar, S. Panda, and A. Perez-Lorenzana, Nucl. Phys. B614, 101 (2001).

[36] M. Majumdar and A.C. Davis, Phys. Rev. D (to be published), hep-th/0304226.

[37] T.W. Kibble, Acta Phys. Pol. B 13, 723 (1982).

[38] W.H. Zurek, Acta Phys. Pol. B 24, 1301 (1993).

[39] D. Mitchell and N. Turok, Phys. Rev. Lett. 58, 1577 (1987); Nucl. Phys. B294, 1138 (1987).

[40] N. Deo, S. Jain, and C.I. Tan, Phys. Rev. D 40, 2626 (1989).

[41] J.J. Atick and E. Witten, Nucl. Phys. B310, 291 (1988).

[42] R. Hagedorn, Nuovo Cimento, Suppl. 3, 147 (1965).

[43] S.A. Abel, J.L. Barbon, I.I. Kogan, and E. Rabinovici, J. High Energy Phys. 04, 015 (1999).

[44] T. Boehm and R. Brandenberger, J. Cosmol. Astropart. Phys. 06, 008 (2003).

[45] R. Easther, B.R. Greene, M.G. Jackson, and D. Kabat, Phys. Rev. D 67, 123501 (2003). 
[46] D. Seckel, Prepared for Inner Space/Outer Space: Conference on Physics at the Interface of Astrophysics/Cosmology and Particle Physics, Batavia, Illinois, 1984.

[47] C.A. Terrero-Escalante, Rev. Mex. Fis. 49S2, 118 (2003)

[48] D.N. Spergel et al., Astrophys. J., Suppl. 148, 175 (2003).

[49] N. Turok and R.H. Brandenberger, Phys. Rev. D 33, 2175 (1986).

[50] V. Bozza, M. Gasperini, M. Giovannini, and G. Veneziano, Phys. Lett. B 543, 14 (2002).

[51] M. Gasperini and G. Veneziano, Astropart. Phys. 1, 317 (1993).

[52] R. Brustein, M. Gasperini, M. Giovannini, V.F. Mukhanov, and G. Veneziano, Phys. Rev. D 51, 6744 (1995).

[53] S. Mollerach, Phys. Rev. D 42, 313 (1990).
[54] D.H. Lyth and D. Wands, Phys. Lett. B 524, 5 (2002).

[55] K. Enqvist and M.S. Sloth, Nucl. Phys. B626, 395 (2002).

[56] T. Moroi and T. Takahashi, Phys. Lett. B 522, 215 (2001); 539, 303(E) (2002).

[57] R. Easther, B.R. Greene, and M.G. Jackson, Phys. Rev. D 66, 023502 (2002).

[58] D.A. Easson, Int. J. Mod. Phys. A 18, 4295 (2003).

[59] S.H. Alexander, J. High Energy Phys. 10, 013 (2003).

[60] N. Kaiser and A. Stebbins, Nature (London) 310, 391 (1984).

[61] R. Durrer, M. Kunz, and A. Melchiorri, Phys. Rep. 364, 1 (2002).

[62] M. Axenides and L. Perivolaropoulos, Phys. Rev. D 56, 1973 (1997); M. Axenides, L. Perivolaropoulos, and M. Trodden, ibid. 58, 083505 (1998). 